\title{
Enumeration of specimens from Edinburgh expedition to northern Sikkim deposited at Sikkim State Forest Herbarium (SSFH): Part - I
}

\author{
D. K. Pradhan
}

Quality Control Laboratory-HARC- Sikkim State Forest Herbarium (SSFH), Forests and Environment Department, Govt. of Sikkim, Deorali-777102, Gangtok, Sikkim, India

E-mail: pradhansikkim@gmail.com

[Received 09.12.2020; Revised 17.12.2020; Accepted 24.12.2020; Published 31.12.2020]

\begin{abstract}
The specimens of Edinburgh Expedition to the Northern Sikkim deposited at Sikkim State Forest Herbarium (SSFH) are enumerated here for future reference. A total of 99 species under 45 genera with their distribution data is presented. Based on the representative specimens of the expedition, the new distributional data of Crassocephalum crepidioides (Benth.) S. Moore, Carex cruciata Wahlenb., Carex atrata L. var. atrata, Carex anomoea Hand.-Mazz. and Carex speciosa Kunth ssp. speciosa were recorded and presented in this paper for reference. These were the new records for the Sikkim Himalaya.
\end{abstract}

Key words: Endemic species, New distribution, Plant diversity, Sikkim Himalaya

\section{INTRODUCTION}

The Sikkim State Forest Herbarium initiated way back in 1978 and obtained the approval of Cabinet of State Government on $14^{\text {th }}$ March, 1978. This was the humble beginning of the Sikkim State Forest Herbarium. The acronym of the Sikkim State Forest Herbarium is SSFH and it houses collections of various distinguished scientists of the world including the collections of the extension work of H. Hara under the guidance of Prof. H. Ohba. The Sikkim State Forest Herbarium institutionalized under the notification 184/ GOS/FED/ Secy dated 9.11.2020 and presently, catalogues and prepares in house database of the biodiversity of Eastern Himalayas involving in several other ancillary activities. The altitudinal gradient of Sikkim is the critical factor which is responsible for the rich plant diversity and endemism in Sikkim Himalaya. Thus, the study of the distribution of the species and genera in this region is significant. The paper communication attempts to document the herbarium specimens deposited by the noted researchers in the Sikkim State Forest Herbarium. The enumerated species were the collection of D.G Long and H.J. Noltie during the Edinburgh Expedition to Northern Sikkim in 1996.

In regard to the expeditions of Sikkim Himalaya, there were many researchers who conducted several works in the flora (Hooker \& Thomson 1855; Hooker 1872-1897; Griffith 1847, 1848; Hara 1964; Grierson \& Long 1983; Ikeda et al. 2008; Maity et al. 2018). Joseph D. Hooker explored Sikkim in 1848 to 1849, subsequently other eminent scientists like C.B. Clarke, J.S. Gamble, H. Hara to name a few. The monumental works of Hooker guided the researchers to some extend to classify the resources. Not only was this but the contribution of W.W. Smith who made several trips to Sikkim along with G.H. Cave is also praiseworthy. These resulted in several publications of the Sikkim Himalaya. At the same time, the native Lepcha collectors Ribu and Rohmoo collected important specimens from Sikkim for the Royal Botanic Garden, Calcutta (now AJC Indian Botanic Garden) in 1911 and all these specimens are deposited at Central National Herbarium (CAL). Further N. L. Bor made extensive collections of grasses in 
Sikkim and the Chumbi valley (Bor 1940, 1960). Recently, the teams led by Hara and Ohba of Tokyo University had also conducted the appreciative works for strengthening the knowledge of Sikkim flora (Hara 1966, 1971; Ohashi 1975; Ikeda et al. 2008).

This paper deals with the collections of Edinburgh expedition to Sikkim, led by D.G. Long and H. J. Noltie of the Royal Botanic Garden, Edinburgh. In regard to the Edinburgh expeditions to Sikkim, there were two expeditions in 1992 to the west and in 1996 to the north (Noltie 1996, 2000). The Sikkim Forest Department received the collections with some type specimens pertaining to the expedition (Noltie 2000). Long and Noltie discovered several new taxa in the region which were the additional information to the phytodiversity of Sikkim. The new distributional data of Crassocephalum crepidioides (Benth.) S. Moore, Carex cruciata Wahlenb. Carex atrata L. var. atrata, Carex anomoea Hand.-Mazz. and Carex speciosa Kunth subsp. speciosa were the new records for the Sikkim Himalaya based on the specimens of Edinburgh expedition.

ENUMERATION OF DEPOSITED HERBARIUM SPECIMENS OF D.G. LONG AND H.J. NOLTIE IN SIKKIM STATE FOREST HERBARIUM (SSFH)

Aphragmus oxycarpus (Hook.f. \& Thomson) Jafri, Notes Roy. Bot. Gard. Edinburgh 22: 96. 1956. [Brassicaceae]

India: Sikkim: North District: Lakes on SE side of Sebu La. Sandy soil by lake. 5040; $27^{\circ} 55^{\prime} \mathrm{N}$, 88³9' E; Cushion-forming; flowers white, anthers yellow; Voucher No. 397; Coll. D. G. Long \& H. J. Noltie

Anaphalis triplinervis (Sims) C.B. Clarke var. monocephala (DC.) Airy Shaw, Bot. Mag. 158: t. 9396. 1935. [Asteraceae]

India: Sikkim: North District: Between Goichang and Club Hut, Lasha Chhu. Sandy shigle by river;2755’50" N, 88 36’52" E; Capitula silvery-white. 4470 m; Voucher No. 356; 20 Jul 1996; Coll. D.G. Long \& H.J. Noltie

Anaphalis triplinervis (Sims) C.B. Clarke var. monocephala (DC.) Airy Shaw, Bot. Mag. 158: t. 9396. 1935. [Asteraceae]

India: Sikkim: North District: Tembawa Chhu valley, E of Yume Samdong. Loose scree. 4735m; Leaves silver; phyllaries white, florets yellow; Voucher No. 425; 24 Jul 1996; Coll. D.G. Long \& H.J. Noltie

Anaphalis xylorhiza Sch.Bip. ex Hook.f., Fl. Brit. India [J. D. Hooker] 3(8): 281. 1881. [Asteraceae] India: Sikkim: North District: Goichang, Lasha Chhu valley. Short grazed turf on slope. 4555 m; $27^{\circ} 5525222$ N, 88 36’17" E; Phyllaries pinkish; Voucher No. 348; 19 Jul 1996; Coll. D.G. Long \& H. J. Noltie

Anaphalis xylorhiza Sch.Bip. ex Hook.f., Fl. Brit. India [J. D. Hooker] 3(8): 281. 1881. [Asteraceae]

India: Sikkim: North District: Tembawa Chhu valley, E of Yume Samdong. Loose scree. 4735 m; $27^{\circ} 54^{\prime}$ N, $88^{\circ} 43^{\prime}$ E; Leaves silver; phyllaries white; Voucher No. 426; Coll. D. G. Long \& H. J. Noltie

Aralia cachemirica Decne., Voy. Inde [Jacquemont] 4(Bot.): 72, t. 81. 1841. [Araliaceae] India: Sikkim: North District: Between Phune and Yakche, $\mathrm{N}$ of Lachung. Bank at edge of Abies forest. $3310 \mathrm{~m} ; 2^{\circ} 44^{\prime} 21^{\prime \prime} \mathrm{N}, 88^{\circ} 44^{\prime} 24^{\prime \prime} \mathrm{E}$; Coarse herb $1.2 \mathrm{~m}$, leaves 3x pinnate; flowers greenish; Voucher No. 199; Coll. D. G. Long \& H.J. Noltie 
Arisaema echinatum (Wall.) Schott, Melet. Bot. 1. 17. 1832. [Araceae]

India: Sikkim: North District: Between Phune and Yakche, $\mathrm{N}$ of Lachung. Short turf beside road on open hillside. $3080 \mathrm{~m} ; 27^{\circ} 43^{\prime} 11^{\prime \prime} \mathrm{N}, 88^{\circ} 45^{\prime} 07^{\prime \prime E} \mathrm{E}$ Spathe tube: outside greenish brown, faintly striped; inside striped purple/red and white. Blade yellowish green, marked purple red at apex, narrow recurved auricles in lower half Spadix sterile, appendix swollen, striped purplish, truncate at base apex rounded, ciliate Voucher No. 214; 14 Jul 1996; Coll. D. G. Long \& H. J. Noltie

Arisaema jacquemontii Blume, Rumphia 1: 95. 1836. [Araceae]

India: Sikkim: North District: Yumthang, Lachung Chhu. Edge of yak pasture in area with boulders. $3600 \mathrm{~m} ; 27^{\circ} 48^{\prime} 08^{\prime \prime} \mathrm{N}, 88^{\circ} 42^{\prime} 22^{\prime \prime} \mathrm{E}$; Spathe green striped white, tinged purplish towards base, recurved portion of spadix appendage brownish; 13 Jul 1996; Voucher No 172; Coll. D. G. Long \& H. J. Noltie

Aristolochia griffithii Hook. f. \& Thomson ex Duch., Prodr. [A.P. de Candolle] 15(1): 437. 1864.. [Aristolochiaceae]

India: Sikkim: North District: Bicchu, S of Lachung; $27^{\circ} 40^{\prime} 08^{\prime \prime} \mathrm{N}, 88^{\circ} 44^{\prime} 14^{\prime \prime}$ E Extensive climber; fruits 6-angled; Scrub covered bank by road. 2650 m; Voucher No. 248; 16 Jul 1996; Coll. D. G. Long \& H. J. Noltie

Aster albescens (DC.) Wall. ex Hand.-Mazz., Acta Horti Gothob. 12: 205. 1938. [Asteraceae] India: Sikkim: North District: Between Yakche and Lachung. Edge of bank by roadside. 3045 $\mathrm{m} ; 27^{\circ} 43^{\prime} 11^{\prime \prime} \mathrm{N}, 88^{\circ} 45^{\prime} 07^{\prime \prime} \mathrm{E}$; Sprawling shrub to $2 \mathrm{~m}$; ligules lilac, disc florets yellow; Voucher No. 231; 15 Jul 1996; Coll. D. G. Long \& H. J. Noltie

Aster asteroides (DC.) Kuntze, Revis. Gen. Pl. 1: 315. 1891. [Asteraceae]

India: Sikkim: North District: Upper Lasha Chhu valley, below Yulhe Khang glacier. Short grazed grass by river. $4545 \mathrm{~m}$. $27^{\circ} 56^{\prime} \mathrm{N}, 88^{\circ} 38^{\prime}$ ' $\mathrm{E}$; Growing singly; roots tuberous; ligules mauve, disc florets yellow; Voucher No. 362; 20 Jul 1996; Coll. D. G. Long \& H. J. Noltie

Blysmus compressus (L.) Panz. ex Link, Hort. Berol. [Link] 1: 278. 1827. [Cyperaceae]

India: Sikkim: North District: Yumthang, Lachung Chhu. Artificial gravel bank in yak pasture. 3600 m; $27^{\circ} 48208^{\prime \prime}$ N, 8842' 22" E; Rhizomatous sedge; Voucher No. 164; 13 Jul 1996; Coll. D. G. Long \& H. J. Noltie

Carex alatauensis S. R. Zhang, Bot. J. Linn. Soc. 179: 19. 2015. [Cyperaceae]

India: Sikkim: North District: Lasha Chhu valley, NE of Thanggu. Short turf on open slope. 3980 m; 2753' 39" N, 88 32' 44" E; Voucher No. 325; 18 Jul 1996; Coll. D. G. Long \& H. J. Noltie

Carex anomoea Hand.-Mazz., Symb. Sin. Pt. VII. 1267. 1936. [Cyperaceae]

India: Sikkim: North District: Bop, between Chungthang and Maltin. Very wet rock beside

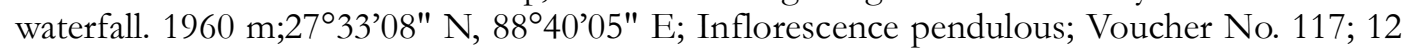
Jul 1996; Coll. D.G. Long \& H.J. Noltie

Carex atrata L., Sp. Pl.: 976. 1753. var. atrata [Cyperaceae]

India: Sikkim: North District: SE side of Lasha Chhu, below Sebe La. Steepslope. 4560 m; Spikes nodding, blackish; Voucher No. 374; Coll. D.G Long \& H. J. Noltie

Carex atrata subsp. pullata (Boott) Kük., Pflanzenr. (Engler) IV. 20(Heft 38): 400. 1909. [Cyperaceae] 
India: Sikkim: North District: SE side of Lasha Chhu, below Sebu La. Steep, grazed slope. 4560 m; 2756’13 “ N, 88 38’30" E; Spikes semi-erect, blackish. Growing with Carex duthiei; Voucher No. 375; 21 Jul 1996; Coll. D. G. Long \& H.J. Noltie

Carex atrofusca subsp. minor (Boott) T. Koyama, Fl. E. Himalaya 3rd. Rep.: 122. 1975. [Cyperaceae]

India: Sikkim: North District: SE side of Sha Chhu, below Sebu La. Steep, grazed 4560 m; Voucher No. 373; 21 Jul 1996; Coll. D. G. Long \& H. J. Noltie

Carex bhutanensis S. R. Zhang, Bot. J. Linn. Soc. 179: 19. 2015. [Cyperaceae]

India: Sikkim: North District: Lasha Chhu valley, NE of Thanggu. Open, grazed turf.4330 m; $27^{\circ} 54^{\prime} 28^{\prime \prime} \mathrm{N}, 88^{\circ} 34^{\prime} 02^{\prime \prime} \mathrm{E}$; Male plants greatly out numbering female; Voucher No. 328; 18 Jul 1996; Coll. D. G. Long \& H. J. Noltie

Carex bonatiana (Kük.) N. A.Ivanova, Bot. Zhurn. S.S.S.R. 24: 501, in obs. 1939. [Cyperaceae] India: Sikkim: North District: Chhoptha, above Thanggu. Open slope. 3980 m; 27 $54^{\prime} 21^{\prime \prime} \mathrm{N}$, 88³2' 04" E; Tufted sedge; some culms erect, other scurved; Voucher No. 295; 17 Jul 1996; Coll. D. G. Long \& H. J. Noltie

Carex bonatiana (Kük.) N. A. Ivanova, Bot. Zhurn. S.S.S.R. 24: 501, in obs. 1939. [Cyperaceae] India: Sikkim: East District: Tsomgo Chho. Yak-grazed slopes above lake. 3720 m;27²2’14 “ N, 88 45'52" E

Densely tufted; Voucher No. 53; 8 Jul 1996; Coll. D. G. Long \& H. J. Noltie

Carex bonatiana (Kük.) N.A.Ivanova, Bot. Zhurn. S.S.S.R. 24: 501, in obs. 1939. [Cyperaceae] India: Sikkim: North District: Yumthang, Lachung Chhu. Artificial gravel bank in yak pasture. 3600 m; $27^{\circ} 48^{\prime} 08^{\prime \prime}$ N, $88^{\circ} 42^{\prime} 22^{\prime \prime}$ E; Densely tufted; stems curved; Voucher No. 163; 13 Jul 1996; Coll. D. G. Long \& H. J. Noltie

Carex burttii Noltie, Edinburgh J. Bot. 50(2): 186. 1993. [Cyperaceae]

India: Sikkim: East District: Rate Chu; bridge, N of Gangtok. Wet cliffs. 1770 m; $27^{\circ} 23^{\prime} 11^{\prime \prime}$ N, 88³8'38" E; Tufted sedge; Voucher No. 90; Coll. D. G. Long \& H. J. Noltie

Carex cercostachys Franch., in Bull. Annuel Soc. Philom. Paris, sér. 8, 7: 27. 1895. [Cyperaceae]

India: Sikkim: North District: Upper Lasha Chhu valley, below Yulhe Khang glacier. Among scrub by river. 4545 m; $27^{\circ} 562$ N 88³8' E; Voucher No. 361; 20 Jul 1996; Coll. D. G. Long \& H. J. Noltie

Carex cercostachys Franch., in Bull. Annuel Soc. Philom. Paris, sér. 8, 7:27. 1895. [Cyperaceae] India: Sikkim: North District: NW slopes above Sebu Chho. Eroded gully in grazed slope.

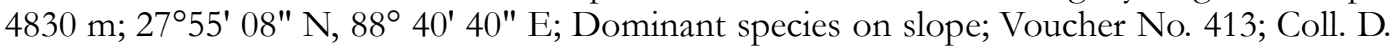
G. Long \& H. J. Noltie

Carex cruciata Wahlenb., in Kongl. Vetensk. Acad. Nya Handl. 24: 149. 1803. [Cyperaceae] India: Sikkim: North District: N of Myang Chhu, S side of Tista Valley. Among scrub on bank by road through broadleaved forest; $1615 \mathrm{~m} ; 27^{\circ} 31^{\prime} \mathrm{N}, 88^{\circ} 36^{\prime} \mathrm{E}$; Utricles pale green (not whitish), maleglumes orange-brown; Voucher No. 113; 11 Jul 1996; Coll. D. G. Long \& H. J. Noltie

Carex curticeps C.B.Clarke, Fl. Brit. India [J. D. Hooker] 6(20): 729. 1894. [Cyperaceae] 
Specimens of Edinburgh expedition to northern Sikkim

India: Sikkim: East District: Above Kyangnosla. Damp, deforested slope. 3530 m;27²2 02" N, 88 42’38" E; Young infl. female, becoming brown; Voucher No. 73; 8 Jul 1996; Coll. D. G. Long \& H. J. Noltie

Carex daltonii Boott, Ill. Gen. Carex 1: 5. 1858. [Cyperaceae]

India: Sikkim: North District: Bop, between Chungthang and Maltin. Wet cliff ledges; 2733'08" N, 8840’05" E; Densely tufted; Voucher No. 119; 12 Jul 1996; Coll. D. G. Long \& H.J. Noltie

Carex deasyi (C.B. Clarke) O.Yano \& S.R. Zhang, Bot. J. Linn. Soc. 179: 20. 2015. [Cyperaceae] India: Sikkim: North District: Chhoptha, above Thanggu. Ungrazed meadow. $3880 \mathrm{~m}$; $27^{\circ} 54^{\prime} 21^{\prime \prime}$ N, $88^{\circ} 32^{\prime}$ 04" E; Densely tufted; culms and leaves glaucous; Voucher No. 302; 17 Jul 1996; Coll. D.G. Long \& H.J. Noltie

Carex decora Boott, Ill. Gen. Carex 1: 5, t. 15. 1858. [Cyperaceae]

India: Sikkim: East District: Below Kyangnosla. Wet slope in deforested area (formerly Fir/ Juniper). 3120 m; $27^{\circ} 22^{\prime}$ N, $88^{\circ} 42^{\prime}$ E; Voucher No. 75; 8 Jul 1996; Coll. D. G. Long \& H. J. Noltie

Carex aff. duthiei C. B. Clarke [Cyperaceae]

India: Sikkim: North District: Lakes on SE side of Sebu La. Flat, wet area by pool. 5040 m; Densely tufted, culms arching; spikes nodding, black; Voucher No. 410; 23 Jul 1996; Coll. D.G. Long \& H.J. Noltie

Carex esenbeckii Kunth, Enum. Pl. [Kunth] 2: 522. 1837. [Cyperaceae]

India: Sikkim: East District: Tsomgo Chho. Yak-grazed slopes above lake. 3720 m; 27²2’14 “ $\mathrm{N}, 88^{\circ} 45^{\prime} 52^{\prime \prime} \mathrm{E}$;

Densely tufted; infl. dark brown; Voucher No. 59;8 Jul 1996; Coll. D. G. Long \& H. J. Noltie

Carex filicina Nees in R.Wight, Contr. Bot. India: 123. 1834. [Cyperaceae]

India: Sikkim: North District: Between Phune and Yakche, $\mathrm{N}$ of Lachung. Bank at edge of Abies forest. 3310 m; 2744’21" N, 8844’24" E; Tufted sedge; Voucher No. 202; 14 Jul 1996; Coll. D. G. Long \& H. J. Noltie

Carex gracilenta Boott ex Boeckeler, Linnaea 41(2): 185. 1877. [Cyperaceae]

India: Sikkim: North District: S of Thanggu. Stony slope among scrub. $3760 \mathrm{~m} ; 27^{\circ} 52^{\prime} 28^{\prime \prime} \mathrm{N}$, 8832 '31" E; Tufted sedge; spikes black, nodding; Voucher No. 275; 17 Jul 1996; Coll. D.G. Long \& H. J. Noltie

Carex gracilenta Boott ex Boeckeler, Linnaea 41(2): 185. 1877. [Cyperaceae]

India: Sikkim: North District: S of Thanggu, among scrub. 3760 m; $27^{\circ} 52^{\prime} 28^{\prime \prime} \mathrm{N}, 88$ 32' 31' E; Spikes green and black, some erect; Voucher No. 279; 17 Jul 1996; Coll. D. G. Long \& H. J. Noltie

Carex haematostoma Nees in R.Wight, Contr. Bot. India: 125 .1834. [Cyperaceae]

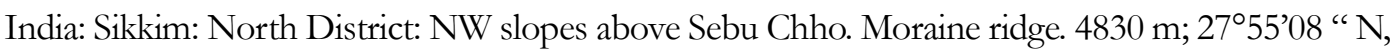
8840’40" E; Spikes blackish, erect; Voucher No. 415; 23 Jul 1996; Coll. D. G. Long \& H. J. Noltie

Carex harae (Rajbh. \&H. Ohba) O. Yano, Bot. J. Linn. Soc. 179: 22. 2015. [Cyperaceae]

India: Sikkim: North District: Phune, Lachung Chhu. On mossy, rotten Abies log in yak pasture.

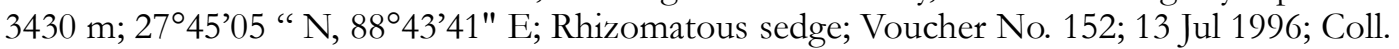
D.G. Long \& H. J. Noltie 
Carex harae (Rajbh. \& H.Ohba) O.Yano, Bot. J. Linn. Soc. 179: 22. 2015. [Cyperaceae] India: Sikkim: North District: Yumthang, Lachung Chhu. Artificial gravel bank in yak pasture, 3600 m; Rhizomatous sedge; Voucher No. 162; 13 Jul 1996; Coll, D.G. Long \& H. J. Noltie

Carex inclinis C.B. Clarke in J.D. Hooker Fl. Brit. India 6(20): 728 1894. [Cyperaceae] India: Sikkim: North District: Between Yakche and Lachung, Edge of bank by roadside. 3045 m; 27²3’11" N, 8845’07" E; Voucher No. 230; 15 Jul 1996; Coll. D. G. Long \& H. J. Noltie

Carex laeta Boott, Ill. Gen. Carex 1: 69. 1858. [Cyperaceae]

India: Sikkim: North District: Chhoptha, above Thanggu. Open healthy slope. 3980 m; 27²5'21" $\mathrm{N}, 88^{\circ} 32^{\prime} 04^{\prime \prime} \mathrm{E}$

Densely tufted sedge; culms slender, curved; Voucher No. 290; 17 Jul 1996; Coll. D. G. Long \& H. J. Noltie

Carex lehmannii Drejer in Symb. Caric.: 13. 1844. [Cyperaceae]

India: Sikkim: North District: Glacial valley above Yakche, $\mathrm{N}$ of Lachung. Moraine patches among scrub at foot of wet cliffs. $3180 \mathrm{~m} ; 27^{\circ} 43^{\prime} 09^{\prime \prime} \mathrm{N}, 88^{\circ} 44^{\prime} 47^{\prime}$ ” E; Tufted sedge; spikelets whitish green; Voucher No. 225; 15 Jul 1996; Coll. D. G. Long \& H. J. Noltie

Carex longipes D.Don ex Tilloch \& Taylor, Philos. Mag. J. 62: 455. 1823. [Cyperaceae]

India: Sikkim: North District: Lema, S of Lachung. Grassy place at edge of path through scrub. 2400 m; 27³8' 24" N, 8843’18 “ E; Utricles green; Voucher No. 126; 12 Jul 1996; Coll. D.G. Long \& H. J. Noltie

Carex munda Boott, Ill. Gen. Carex 1: 7. 1858. [Cyperaceae]

India: Sikkim: North District: Phune, Lachung Chhu. On mossy log in Abies forest. $3430 \mathrm{~m}$; Tufted sedge; Voucher No. 141; Coll. D.G. Long \& H. J. Noltie

Carex munda Boott, Ill. Gen. Carex 1: 7. 1858. [Cyperaceae]

India: Sikkim: North District: S of Thanggu Open stony slope by road. $3760 \mathrm{~m} ; 27^{\circ} 52^{\prime} 28^{\text {“ }} \mathrm{N}$, 88³2’31" E; Voucher No. 277; 17 Jul 1996; Coll. D.G. Long \& H.J. Noltie

Carex parvula O.Yano, Bot. J. Linn. Soc. 179: 24. 2015. [Cyperaceae]

India: Sikkim: North District: NW above Sebu Chho Grazed slope. $4830 \mathrm{~m} ; 27^{\circ} 55^{\prime} 08^{\prime \prime} \mathrm{N}$, 8840’40" E; Mat-forming; plants entirely male; Voucher No. 411; 23 Jul 1996; Coll. D.G. Long \& H.J. Noltie

Carex pseudofoetida Kük. in Ostenf., Bot. Tidsskr. 28: 226. 1907. [Cyperaceae]

India: Sikkim: North District: Upper Lasha Chhu valley, below Yulhe Khang glacier. Marshy, sandy area near river. 4545 m; $27^{\circ} 56^{\prime} \mathrm{N}, 88^{\circ} 38^{\prime}$ E; Rhizomatous sedge; Voucher No. 364; 20 Jul 1996; Coll. D. G. Long \& H. J. Noltie

Carex pseudolaxa (C.B. Clarke) O. Yano \& S.R. Zhang, Bot. J. Linn. Soc. 179: 24. 2015. [Cyperaceae]

India: Sikkim: North District: Yakche, N of Lachung. Gravelly marsh. 3105 m; $27^{\circ} 43^{\prime} 17^{\prime \prime} \mathrm{N}$, $88^{\circ} 45^{\prime}$ 02" E; Young infls. female, older ones male at apex; Voucher No. 211; 14 Jul 1996; Coll. D.G. Long \& H. J. Noltie

Carex pseudolaxa (C.B.Clarke) O.Yano \& S.R.Zhang, Bot. J. Linn. Soc. 179: 24. 2015. [Cyperaceae] 
India: Sikkim: North District: Glacial valley above Yakche, $\mathrm{N}$ of Lachung. On rocks and moraine. $3180 \mathrm{~m} ; 27^{\circ} 43^{\prime} 09^{\prime \prime} \mathrm{N}, 88^{\circ} 44^{\prime} 47^{\prime \prime} \mathrm{E}$; Shortly rhizomatous, spikelets dark brown; Voucher No. 228; Coll. D.G. Long \& H.J. Noltie

Carex rara Boott, Trans. Linn. Soc. London 20(1): 139. 1846. [Cyperaceae]

India: Sikkim: North District: Phune, Lachung Chhu. Wet yak pasture cleared from Abies

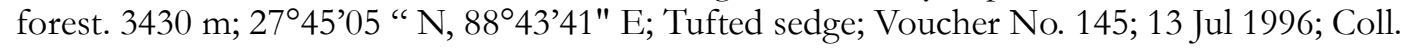
D.G. Long \& H. J. Noltie

Carex rochebrunei Franch. \& Sav., Enum. Pl. Jap. 2: 555. 1878. [Cyperaceae]

India: Sikkim: North District: Phune, Lachung Chhu. Abies forest. 3430 m;2745’05" N, 8843'41" E; Tufted sedge.

Voucher No. 140; 13 Jul 1996; Coll. D.G. Long \& H. J. Noltie

Carex speciosa Kunth in Enum. Pl. 2: 504. 1837. subsp.speciosa [Cyperaceae]

India: Sikkim: East District: Namnam area, SE outskirts of Gangtok. Degraded Schima forest in deep shade. $1730 \mathrm{~m} ; 2719^{\prime} \mathrm{N}, 8^{\circ} 36^{\prime} \mathrm{E}$; Utricles green, male part of infl. Whitish; Voucher No. 26; 6 Jul 1996; Coll. D.G. Long \& H.J. Noltie

Carex unciniiformis Boeckeler, in Beitr. Cyper. 1: 40. 1888. [Cyperaceae]

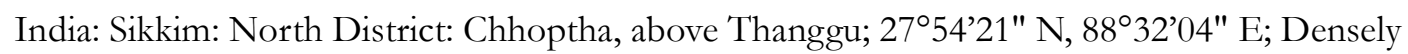
tufted; Open healthy slope. 3980 m; Voucher No. 291; 17 Jul 1996; Coll. D. G. Long \& H. J. Noltie

Carex uncinioides Boott, Ill. Gen. Carex 1: 8. 1858. [Cyperaceae]

India: Sikkim: North District: SE side of Lasha Chhu, below Sebu La. Steep, grazed slope. 4560 m; 2756’13" N, 88³8’30" E; Voucher No. 372; 21 Jul 1996; Coll. D.G. Long \& H.J. Noltie

Carex uncinioides Boott, Ill. Gen. Carex 1: 8. 1858. [Cyperaceae] India: Sikkim: East District: Tsomgo Chho. Yak-grazed slopes above lake. 3720 m; 27²2’14" N, 88 45’52" E; Infl. greenish brown; Voucher No. 51; 8 Jul 1996; Coll. D.G. Long \& H. J. Noltie

Carex vidua Boott ex C.B.Clarke, in J.D. Hooker, Fl. Brit. India 6(20): 713. 1894. [Cyperaceae] India: Sikkim: North District: Chhoptha, above Thanggu. Open healthy slope. 3980 m; 2754'21" N, 88³2' 04" E; Voucher No. 297, 288; 17 Jul 1996; Coll. D.G. Long \& H.J. Noltie

Carex wallichiana Spreng., Syst. Veg., ed. 16 [Sprengel] 3: 812. 1826. [Cyperaceae]

India: Sikkim: North District: Lachung Army Encampment. Wet rubbish dump among scrub; $27^{\circ} 41^{\prime} \mathrm{N}, 88^{\circ} 45^{\prime}$ E; Densely tufted sege; culms, spikes green; Voucher No. 238; 15 Jul 1996; Coll. D. G. Long \& H. J. Noltie

Carpesium nepalense Less., Linnaea 6(2): 234. 1831. [Asteraceae]

India: Sikkim: North District: Phune, Lachung Chhu. Abies forest (disturbed areas). $3430 \mathrm{~m}$; 2745’05" N, 88 43’41" E; Inflorescences nodding, yellow; Voucher No. 139; 13 Jul 1996; Coll. D. G. Long \& H. J. Noltie

Cassiope fastigiata (Wall.) D. Don, Edinburgh New Philos. J. 17: 156. 1834. [Ericaceae] India: Sikkim: North District: Tembawa Chhu valley, B of Yume Samdong. Grazed slope; $27^{\circ} 54$ ' N 8843' E; Flowers white. Voucher No. 423; 24 Jul 1996; Coll. D. G. Long \& E.J. Noltie 
Chlorophytum nepalense (Lindl.) Baker, J. Linn. Soc., Bot. 15: 330. 1876. [Asparagaceae] India: Sikkim: North District: $\mathrm{N}$ bank of Rate Chu, W of bridge. Among scrub by stream in ravine, forest cleared; $27^{\circ} 23^{\prime} 25^{\prime \prime}$ N, 88 38’32" E; Petals white, anthers yellow; 1730 m; Voucher No. 96; Coll. D.G. Long \& H. J. Noltie

Codonopsis foetens Hook.f. \& Thomson in J. Proc. Linn. Soc., Bot. 2: 16. 1857. [Campanulaceae]

India: Sikkim: North District: Lasha Chhu, below Phaklung. Among scrub and boulders on river bank. $4420 \mathrm{~m} ; 27^{\circ} 55^{\prime} 22^{\prime \prime} \mathrm{N}, 88^{\circ} 35^{\prime} 08^{\prime \prime} \mathrm{E}$; Corolla pale blue, veined purple inside; Voucher No. 336; 19 Jul 1996; Coll. D. G. Long \& H. J. Noltie

Codonopsis gracilis Hook.f. \& Thomson, Illust. Himal. Pl. t. 16 A. 1855. [Campanulaceae] India: Sikkim: North District: Menshithang, NW of Chungthang. Scrub covered bank by road. 1930 m; $27^{\circ} 38^{\prime} \mathrm{N}, 88^{\circ} 37^{\prime}$ E Climbing, foetid herb; flowers pale lilac-blue; Voucher No. 249; Coll. D.G. Long \& H. J. Noltie

Colocasia affinis Schott in Bonplandia 7: 28. 1859. [Araceae]

India: Sikkim: East District: Below Martam, Rongni Chhu Valley. On damp shady cliff. 920m. Spreading by slender stolons; leaves marked purple above, spathe blade oblong 42 × $15 \mathrm{~mm}$, cream, abruptly acuminate, tube green; ovaries cream, upper neuters pink, anthers orange, appendix yellow; Voucher No. 42; 7 Jul 1996; Coll. D. G. Long \& H.J. Noltie

Coriaria terminalis Hemsl. in Hooker's Icon. Pl. 23: t. 2220. 1892. [Coriariaceae]

India: Sikkim: North District: Between Phune and Yakche, N of Lachung. Among shrubs

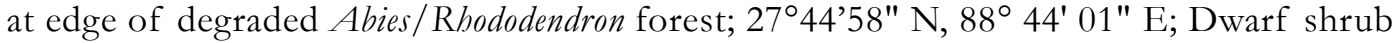
to $50 \mathrm{~cm}$; anthers orange-red. Voucher No. 192, 14 Jul 1996; Coll. D. G. Long \& H. J. Noltie

Crassocephalum crepidioides (Benth.) S. Moore in J. Bot. 50: 211. 1912. [Asteraceae]

India: Sikkim: North District: Rate Chu bridge, N of Gangtok. Roadside. 1770 m; $27^{\circ} 232$ $20^{\prime \prime}$ N, 88³8' 38" E; Disc florets dark orange. Voucher No. 95; 10 Jul 1996; Coll. D. G. Long \& H. J. Noltie

Cremanthodium oblongatum C.B. Clarke, Compos. Ind. 168. 1876. [Asteraceae]

India: Sikkim: North District: NW slopes above Sebu Chho. Grazed slope. 4830 m; 2755’08" $\mathrm{N}, 88^{\circ} 40^{\prime} 40^{\prime \prime} \mathrm{E}$; Leaves rugose above; infls. nodding, phyllaries blackish, ligules pale yellow; anthers dark. Voucher No. 412, 23 Jul 1996; Coll. D. G. Long \& H. J. Noltie

Cremanthodium thomsonii C.B. Clarke, Compos. Ind. 169. 1876. [Asteraceae]

India: Sikkim: North District: SE side of Lasha Chhu, below Sebu La. Steep, grazed slope. 4560 m; 2756’13 “ N, 88³8’30" E; Stems reddish; ligules pale yellow; Voucher No. 369;21 Jul 1996; Coll. D. G. Long \& H. J. Noltie

Cyananthus macrocalyx subsp. spathulifolius (Nannf.) K.K. Shrestha, Acta Phytotax. Sin. 35(5): 412. 1997. [Campanulaceae]

India: Sikkim: North District: Phaklung, Lasha Chhu valley. Hummocky marsh. 4620 m; $27^{\circ} 56^{\prime} 01^{\prime \prime}$ N, 88 35’31" E; Decumbent herb; flowers lemon yellow, tube marked with purple lines on outside; Voucher No. 330; Coll. D.G. Long \& H.J. Noltie

Cynoglossum lanceolatum Forssk.,Fl. Aegypt.-Arab. 41. 1775. [Boraginaceae] 
India: Sikkim: North District: Rate Chu bridge, N of Gangtok. Roadside. 1770 m; 27²3’20" N, 88³8'38" E; Flowers greyish-white, with blue centre; Voucher No. 93; 10 Jul 1996; Coll. D. G. Long \& H. J. Noltie

Cynoglossum wallichii G. Don, Gen. Hist. 4. 354. 1837. [Boraginaceae]

India: Sikkim: North District: Phune, Lachung Chhu. Yak pasture cleared from Abies forest. 3430 m; 2745’05" N, 8843’41" E; Flowers dark blue. Voucher No. 143;13 Jul 1996; Coll. D. G. Long \& H. J. Noltie

Cyperus compressus L. in Biblioth. Bot. 85: 430. 1915. [Cyperaceae]

India: Sikkim: North District: Mangan, Tista Valley. Wet area beside road. 1340 m; 27²9’55" N, 88³2'29" E; Stem bases purplish; spikelets green, very compressed; Voucher No. 106;11 Jul 1996; Coll. D. G. Long \&H. J. Noltie

Cyperus haspan L., Sp. Pl.: 45. 1753. subsp. haspan: Flora Malesiana Series I (1974). [Cyperaceae]

India: Sikkim: East District: Namnam area, SE outskirts of Gangtok, Marshy bank at edge of degraded Schima forest. $1680 \mathrm{~m} ; 27^{\circ} 19^{\prime} \mathrm{N}, 88^{\circ} 36^{\prime} \mathrm{E}$; Tufted sedge; spikelets pale brown; Voucher No. 28; 6 Jul 1996; Coll. D.G. Long \& H.J. Noltie

Cyperus laxus Lam., Tabl. Encycl. 1. 146. 1791. [Cyperaceae]

India: Sikkim: East District: Below Martam, Rongni Chhu Valley. Degraded subtropical vegetation (with scrub and Mikania) in ravine. $920 \mathrm{~m} ; 27^{\circ} 15^{\prime} \mathrm{N}, 88^{\circ} 32^{\prime} \mathrm{E}$; Tufted sedge; Voucher No. 37; 7 Jul 1996; Coll. D. G. Long \& H. J. Noltie

Draba elata Hook.f. \& Thomson in J. Proc. Linn. Soc., Bot. 5: 150. 1860. [Brassicaceae] India: Sikkim: North District: Lasha Chhu, below Phaklung. Rocky gully. 4440 m; 2755’30" N, 88³5’17" E; Flowers yellow; Voucher No. 342;19 Jul 1996; Coll. D. G. Long \& H. J. Noltie

Draba lasiophylla Royle, Ill. Bot. Himal. Mts. [Royle] 71. 1834. [Brassicaceae]

India: Sikkim: North District: Lasha Chhu, below Phaklung. Rocky gully. 4440 m; 2755'30" N, 88³5’17" E; Flowers white; Voucher No. 341; 19 Jul 1996; Coll. D.G. Long \& H.J. Noltie

Eleocharis yunnanensis Svenson in Rhodora 41: 559. 1939. [Cyperaceae]

India: Sikkim: North District: Lachung. Gravelly marsh. 3105 m; 27043’17" N, 8845’02" E; Rhizomatous sedge; Voucher No. 210; Coll. D. G. Long \& H. J. Noltie

Eleutherococcus cissifolius (Griff. ex C.B.Clarke) Nakai in Chosen Shokubutsu 1: 420. 1914. [Araliaceae]

India: Sikkim: North District: Between Phune and Yakche, $\mathrm{N}$ of Lachung. Bank at edge of Abies forest. $3310 \mathrm{~m} ; 27^{\circ} 44^{\prime} 21^{\prime \prime} \mathrm{N}, 88^{\circ} 44^{\prime} 24^{\prime \prime} \mathrm{E}$; Tree to $3 \mathrm{~m}$; fruits green flushed brown Voucher No. 200; 14 Jul 1996; Coll. D.G. Long \& H.J. Noltie

Erigeron multiradiatus (Lindl. ex DC.) Benth. ex C.B.Clarke, Compos. Ind. 56. 1876. [Asteraceae]

India: Sikkim: North District: Yumthang, Lachung Chhu. Grassy yak pasture. $3600 \mathrm{~m} ; 2^{\circ} 48^{\prime}$ $08^{\prime \prime} \mathrm{N}, 88^{\circ} 42^{\prime} 22^{\prime \prime} \mathrm{E}$; Rootstock thick, horizontal, not stoloniferous; ray florets mauve, disc florets yellow; Voucher No. 173; 13 Jul 1996;Coll. D.G. Long \& H.J. Noltie

Erigeron multiradiatus (Lindl. ex DC.) Benth. ex C.B. Clarke, Compos. Ind. 56. 1876. [Asteraceae] 
India: Sikkim: North District: Yume Sam Grazed meadow on slope. $4560 \mathrm{~m} ; 27^{\circ} 54^{\prime} 18^{\prime \prime} \mathrm{N}$, $88^{\circ} 42^{\prime} 5622 \mathrm{E}$; Ligules erect in bud, reddish turning disc florets brown in centre, yellow at edge; Voucher No. 433; 24 July 1996; Coll. D.G. Long \& H.J. Noltie

Eutrema scapiflorum (Hook.f. \& Thomson) Al-Shehbaz, G.Q.Hao \& J.Quan Liu, Bot. J. Linn. Soc. 184(2): 219. 2017. [Brassicaceae]

India: Sikkim: North District: W side Sebu La. In shallow, rocky stream. 492; 27 $55^{\prime} 56^{\prime \prime} \mathrm{N}$, 88³9’01" E; Petals white, tinged grayish;Voucher No. 391; Coll. D. G. Long \& H. J. Noltie

Fimbristylis complanata (Retz.) Link, Hort. Berol. [Link] 1: 292. 1827. [Cyperaceae]

India: Sikkim: North District: E bank of Lachung Chhu, just above Lachung. Grazed turf. 2730 m; $27^{\circ} 41^{\prime} 37^{\prime \prime}$ N, $88^{\circ} 45$ 10" E; Tufted sedge; spikelets brown; Voucher No. 246; 15 Jul 1996; Coll. D. G. Long \& H. J. Noltie

Globba clarkei Baker in J.D. Hooker, Fl. Brit. India 6(17): 210. 1890. [Zingiberaceae]

India: Sikkim: East District: Deorali; Gangtok, Schima woodland, in shade. $1630 \mathrm{~m} ; 27^{\circ} 18^{\prime}$ 28' N, $88^{\circ}$ 36' 35" E; 9 Jul 1996; Coll, D. G. Long \& H. J. Noltie

Hoya acuminata (Wight) Benth. ex Hook.f. in J.D. Hooker, Fl. Brit. India 4(10): 53. 1883. [Apocynaceae]

India: Sikkim: North District: Myang Chhu, S side of Tista Valley. Wet, shady cliffs on moss. $1615 \mathrm{~m} ; 27^{\circ} 31^{\prime} \mathrm{N}, 8836^{\prime} \mathrm{E}$; Pendulous succulent herb; corona pink, petals and pedicels white; Voucher No. 108; 11 Jul 1996; Coll. D.G. Long \& H.J. Noltie

Ilex intricata Hook.f., Fl. Brit. India 1(3): 602. 1875. [Aquifoliaceae]

India: Sikkim: North District: Between and Yakche, $\mathrm{N}$ of Lachung. Among boulders Abies/ Rhododendron forest. $3390 \mathrm{~m}$; $27^{\circ} 45^{\prime} 03^{\prime \prime} \mathrm{N}, 8^{\circ} 43^{\prime} 51^{\prime \prime} \mathrm{E}$; Small shrub to $40 \mathrm{~cm}$; leaves dark green, lossy above, paler beneath; fruits red; Voucher No. 183; 14 Jul 1996; Coll. D. G. Long \& H. J. Noltie

Impatiens decipiens Hook.f. in Rec. Bot. Surv. India 4: 17. 1905. [Balsaminaceae]

India: Sikkim: East District: Below Martam, Rongni chhu Valley. Degraded subtropical vegetation (with scrub and Mikania) in ravine. $880 \mathrm{~m} ; 27^{\circ} 15^{\prime} \mathrm{N}, 88^{\circ} 32^{\prime} \mathrm{E}$; Succulent herb to $1 \mathrm{~m}$; flowers cerise pink, spur slender decurved; Voucher No. 34; 7 Jul 1996; Coll. D.G. Long \& H. J. Noltie

Impatiens racemosa DC., Prodr. [A. P. de Candolle] 1: 688. 1824. [Balsaminaceae]

India: Sikkim: North District: Between Yakche and Lachung. By stream among scrub in degraded

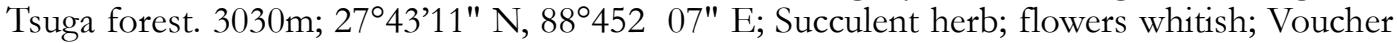
No. 233; 15 Jul 1996; Coll. D.G. Long \& H.J. Noltie

Impatiens stenantha Hook. f., Fl. Brit. India 1(3): 478. 1875. [Balsaminaceae]

India: Sikkim: East District: FambongLho, ridge W of Pangthang Police Camp. Degraded evergreen oak forest; $2130 \mathrm{~m} ; 27^{\circ} 21^{\prime} \mathrm{N}, 88^{\circ} 36^{\prime} \mathrm{E}$; Erect herb; flowers yellow, spotted red on spur. Voucher No. 8; 5 Jul 1996; Coll. D.G. Long \& H.J. Noltie

Isolepis setacea R.Br.,Prodr. Fl. Nov. Holland. 222. 1810. [Cyperaceae]

India: Sikkim: North District: E bank of Lachung Chhu, just above Lachung. Open sandy area at edge of stream. $2730 \mathrm{~m} ; 27^{\circ} 41^{\prime} 37^{\prime \prime} \mathrm{N}, 88^{\circ} 45^{\prime} 10^{\prime \prime} \mathrm{E}$; Tufted sedge; spikelets black; Voucher No. 245; 15 Jul 1996; Coll. D.G. Long \& H.J. Noltie 
Jacobaea raphanifolia (Wall. ex DC.) B. Nord. in Compositae Newslett. 44: 13. 2006. [Asteraceae]

India: Sikkim: East District: Tsongo Chho. Yak-grazed slopes above lake. 3720 m; 27²2’14" $\mathrm{N}, 88^{\circ} 45^{\prime} 52^{\prime \prime} \mathrm{E}$; Biennial; stems purple at base; ray florets dark yellow, phyllaries dark-tipped; Voucher No. 44; 8 Jul 1996; Coll. D. G. Long \& H. J. Noltie

Juniperus pseudosabina Fisch. \& C.A. Mey in Index Seminum (LE, Petropolitanus) 8: 65. 1842. [Cupressaceae]

India: Sikkim: North District: Goichang; Lasha Chhu valley. On scree. 4555 m; 2755' 52" N, 88 36' 17" E; Prostrate shrub; Voucher No. 347; 19 Jul 1996; Coll. D. G. Long \& H. J. Noltie

Ligularia fischeri (Ledeb.) Turcz., Bull. Soc. Imp. Naturalistes Moscou 20(3): 11. 1847. [Asteraceae]

India: Sikkim: North District: E of Lachung Chhu just above Lachung, scrub near river 2730 m; $27^{\circ} 41^{\prime} 37^{\prime \prime} \mathrm{N}, 88^{\circ} 45^{\prime} 10^{\prime \prime} \mathrm{E}$; Herb to 1.lm; ligules yellow, anther purple. Voucher No. 241; Coll. D. G. Long \& H. J. Noltie

Ligularia hookeri f. latiligulatum (R.D. Good) R. Mathur, Fl. Ind. Enum. Asteraceae [R. R. Rao et al.] 53. 1988. [Asteraceae]

India: Sikkim: North District: So on mossy rock among scrub. 3760 m;27² $52^{\prime} 28^{\prime \prime} \mathrm{N}, 88^{\circ} 32^{\prime} 31^{\prime \prime}$ E; Stems purple; phyllaries tinged, Flowers yellow; Voucher No. 278;Coll. D. G. Long \& R. J. Noitie

Ligularia latiligulata (R.D.Good) Spring., Edinburgh J. Bot. 57(3): 402. 2000. [Asteraceae] India: Sikkim: North District: Between Phune and Yakche, $\mathrm{N}$ of Lachung. Abies/Rhododendron forest. $3390 \mathrm{~m}$; $27^{\circ} 45^{\prime} 03^{\prime \prime} \mathrm{N}, 88^{\circ} 43$ '51" E; Ligules slender, yellow, anthers brown; Voucher No. 186; 14 Jul 1996; Coll. D. G. Long \& H. J. Noltie

Lonicera acuminata Wall., Fl. Ind. (Carey \& Wallich ed.) 2: 176. 1824. [Caprifoliaceae] India: Sikkim: North District: Between Phune and Yakche, N of Lachung. Bank beside road on open hillside. $3080 \mathrm{~m} ; 27^{\circ} 43^{\prime} 11^{\prime \prime} \mathrm{N}, 88$ 45’07" E; Shrub to 5m; corolla tube white, marked red, lower lip white with red central stripe, upper lip with 4 lobes, white with 4 red stripes. Voucher No. 215; Coll. D. G. Long \& H. J. Noltie

Lonicera lanceolata Wall., Fl. Ind. (Carey \& Wallich ed.) 2: 177. 1824. [Caprifoliaceae] India: Sikkim: North District: Phune, Lachung Chhu. Abies/Rhodo forest. 3430 m; 27 45’05" N, 8843 41" E; Shrub; flowers dark pink; Voucher No. 178; 13 Jul 1996; Coll. D. G. Long \& H. J. Noltie

Nannoglottis hookeri (Clarke ex Hook.f.) Kitam., Acta Phytotax. Geobot. 31(1-3): 50. 1980. [Asteraceae]

India: Sikkim: North District: $S$ of Thanggu. Degraded slope (formerly fir forest) with Spiraea/ Rosa scrub. 3760 m; 2752'28" N, 8832 '31" E; Herb to 60cm; flowers yellow, anthers brown; Voucher No. 268; 17 Jul 1996; Coll. D. G. Long \& H. J. Noltie

Panax pseudoginseng Wall., Trans. Med. Soc. Calcutta 4: 117. 1829. [Araliaceae] India: Sikkim: North District: Yumthang, Lachung Chhu. Among boulders in Abies forest.

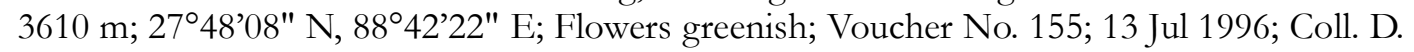
G. Long \& H. J. Noltie 
Rhaphidophora decursiva (Roxb.) Schott in Bonplandia 5: 45. 1857. [Araceae]India: Sikkim: North District: $\mathrm{N}$ bank of Rate Chu, W of bridge. On rocks in scrub cleared of forest in ravine. $1730 \mathrm{~m}$; $27^{\circ} 23^{\prime} 25^{\prime \prime} \mathrm{N}, 88^{\circ} 38^{\prime} 32^{\prime \prime} \mathrm{E}$; Climber; stem c 4cm diameter, leaves bright shining green above, paler beneath. Voucher No. 100; 10 Jul 1996; Coll. D. G. Long \& H. J. Noltie

Rhodiola bupleuroides (Wall. ex Hook. f. \& Thomson) Fu, Acta Phytotax. Sin., Addit. 124. 1965. [Crassulaceae]

India: Sikkim: North District: Glacial valley above Yakche, $\mathrm{N}$ of Lachung. Among scrub at foot of wet cliff; $27^{\circ} 43^{\prime} 09^{\prime \prime} \mathrm{N}, 88^{\circ} 44^{\prime}$ 47" E; Tepals and carpels dark red.3180 m; Voucher No. 227;15 Jul 1996; Coll. D. G. Long \& H. J. Noltie

Rhodiola bupleuroides (Wall. ex Hook.f. \& Thomson) Fu, Acta Phytotax. Sin.,Addit. 124. 1965. [Crassulaceae]

India: Sikkim: North District: Lasha Chhu, below Phaklung. Rocky gully. 4440 m; 2755' 30' N, $88^{\circ} 35^{\prime} 17^{\prime \prime}$ E; Leaves glaucous; young fruits purplish-red; Voucher No. 344; Coll. D. G. Long \& H. J. Noltie

Sarcococca hookeriana Baill., Monog. Bux. 53. 1859. [Buxaceae]

India: Sikkim: North District: Lema, S of Lachung. Rocky bank by road. 2400 m; 27³8'24" N, $88^{\circ} 43^{\prime} 18^{\prime \prime}$ E; Subshrub to 40 cm; fruit green; Voucher No. 122; 12 Jul 1996; Coll. D. G. Long \& H. J. Noltie

Saussurea eriostemon Wall. ex C.B. Clarke, Compos. Ind. 229. 1876. [Asteraceae]

India: Sikkim: Bast District: Tsongo Chho Yak-grazed slopes above lake. 3720 m; 27²2'14" N, 88045'52" E; Phyllaries blackish-purple, tipped green; Voucher No. 45; 27 Jul 1996; Coll. D. G. Long \& H. J. Noltie

Saussurea gossypiphora D.Don, Mem. Wern. Nat. Hist. Soc. 3: 414. 1820. [Asteraceae]

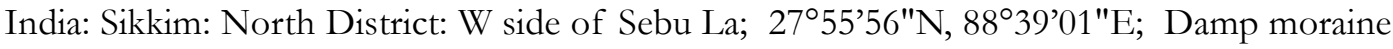
slopes. 4920 m; Voucher No. 389; 21 Jul 1996; Coll. D. G. Long \& H. J. Noltie

Saussurea hieracioides Hook.f., Fl. Brit. India 3(8): 371. 1881. [Asteraceae]

India: Sikkim: North District: Lasha Chhu valley, NE of Thanggu. Among rocks and scrub. 3980 m; $27^{\circ}$ 53' 39" N, 88 32' 44" E; Florets dark bluish purple; Voucher No. 313; 18 Jul 1996; Coll. D. G. Long \& H. J. Noltie

Saussurea hookeri C.B. Clarke, Compos. Ind. 230. 1876. [Asteraceae]

India: Sikkim: North District: Lakes on SE side of Sebu La. Marshy ground by stream. 5040 m; $27^{\circ}$ 55' N 88³9' E; Phyllaries dark purplish; Voucher No. 40222 Jul 1996; Coll. D. G. Long \& H. J. Noltie

Silene baccifera Roth, Tent. Fl. Germ. 1: 192. 1788. [Caryophyllaceae]

India: Sikkim: North District: Lachen, $\mathrm{N}$ edge of village. Open, degraded bank above road.

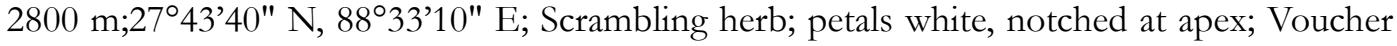
No. 262; 16 Jul 1996; Coll. D. G. Long \& H. J. Noltie

Silene gonosperma (Rupr.) Bocquet, Candollea 22: 7. 1967. [Caryophyllaceae]

India: Sikkim: North District: Upper Lasha Chhu valley, below Yulhe Khang glacier. Among scrub by river. $4545 \mathrm{~m} ; 27^{\circ} 56^{\prime} \mathrm{N}, 88^{\circ} 38^{\prime} \mathrm{E}$; Flowers nodding, calyx veined blackish, petals brownish outside, whitish inside. Voucher No. 357; 20 Jul 19; Coll. D. G. Long \& H. J. Noltie 
Sonchus wightianus subsp. wallichianus (DC.) Boulos, Bot. Not. 125(4): 297. 1972. [Asteraceae]

India: Sikkim: East District: Rate Chu bridge, $\mathrm{N}$ of Gangtok. Cervices in wall by road. 1770 $\mathrm{m} ; 27^{\circ} 23^{\prime} 11^{\prime \prime} \mathrm{N}, 88^{\circ} 38^{\prime} 38^{\prime \prime} \mathrm{E}$; Leaves glaucous beneath; ray florets orange outside, yellow inside. Voucher No. 91;10 Jul 1996; Coll. D. G. Long \& H. J. Noltie

Soroseris hookeriana Stebbins in Mem: Torrey Bot. Club 19(3): 45. 1940. [Asteraceae]

India: Sikkim: North District: SE side of Lasha Chhu, below Sebu La. Steep, grazed slope. $4560 \mathrm{~m} ; 2^{\circ} 56^{\prime} 13^{\prime \prime} \mathrm{N}, 88^{\circ} 38^{\prime} 30^{\prime \prime} \mathrm{E}$; Flower buds dark brown, ligules pale yellow; Voucher No. 370; 21 Jul 1996; Coll. D. G. Long \& H. J. Noltie

Stellaria congestiflora H. Hara in J. Jap. Bot. 52(7): 195. 1977. [Caryophyllaceae]

India: Sikkim: North District: Lasha Chhu, below Phaklung. Rocky gully. 4440 m; 2755’30" N, 88 35’172 2 E; Petals white, deeply 2-lobed, lobes linear. Voucher No. 343; 19 Jul 1996; Coll. D. G. Long \& H. J. Noltie

Strobilanthes wallichii Nees in N.Wallich, Pl. Asiat. Rar. 3: 87. 1832. [Acanthaceae]

India: Sikkim: North District: Glacial valley above Yakche, $\mathrm{N}$ of Lachung. Scrub at foot of wet cliff. 3180 m; Corolla lilac-blue, tube white; Voucher No. 219; Coll. D. G. Long \& H. J. Noltie

Taraxacum sect. Tibetana Soest. [Asteraceae]

India: Sikkim: North District: Goichang, Lasha Chhu valley. Grassland around old settlement. 4480 m; $27^{\circ} 55^{\prime} 52^{\prime \prime} \mathrm{N}, 88^{\circ} 36^{\prime} 17^{\prime \prime} \mathrm{E}$; Ligules cream, the outer brownish-pink on outside; Voucher No. 354;20 Jul 1996; Coll. D. G. Long \& H. J. Noltie

\section{DISCUSSION}

The Eastern Himalayan region has been explored by many researchers and the flora and fauna recorded are not only unique, but also many of them are rare and threatened (Griffith 1847, 1848; Hara 1964; Grierson \& Long 1983; Ikeda et al. 2008; Maity et al. 2018). The Government of Sikkim is formally recording the flora and fauna, and the representative species of the region and the collected specimens have been deposited in the Sikkim State Forest Herbarium (SSFH). It has the records of many representative species of the distinguished scientists and explorers of the world. Due to anthropogenic activities and climate change, several plant species are migrating. The 'Migration lag' is an upcoming issue which is threatening the biodiversity (Corlet \& Westcott 2013). Hence, the recording of the vegetation distribution in the altitudinal gradients of Sikkim Himalaya is needed as it is one of the biodiversity hotspots of the world.

\section{Acknowledgements}

Author is thankful to the Department of Forest and Environment, Government of Sikkim for the kind support. Author thanks to Dr H. J. Noltie for helping and sharing the report to improve the content. The sincere thanks to Prof A.P. Das, formerly of North Bengal University, and Dr. D. Maity, Calcutta University, for their encouragement and supports. He is also thankful to Dr. Shruti Kasana for her assistance.

\section{LITERATURE CITED}

Bor, N.L. 1940. Flora of Assam, Vol. 5. Gramineae, Government of Assam, Calcutta.

Bor, N.L. 1960. The Grasses of Burma, Ceylon, India and Pakistan (excluding Bambuseae). International Book Distributors, Dehradun, Uttarkhand, India. Pp 1-767. 
Corlet, R.T \& Westcott, D.A 2013. Will plant movements keep up with climate change? Trends in Ecology and Evolution 28 (8): 482 - 488

Grierson, A.J.C. \& Long, D.G. 1983. Flora of Bhutan. Vol 1(1), Edinburgh, Royal Botanic Garden, Edinburgh.

Griffith, W. 1847. Journals of Travels in Assam, Burma, Bootan, Affghanistan and the Neighbouring Countries. Vol. 1, Bishop's College Press, Calcutta.

Griffith, W. 1848. Itinerary Notes of plants collected in the Khasyah and Bootan Mountains, 183 7-8, in Affghanisthan and Neighbouring Countries 1839 to 1841. Vol 2, John M'Clelland, F.L.S., J.F. Bellamy, Calcutta.

Hara, H. 1966. The Flora of Eastern Himalaya. First Report, University of Tokyo, Japan.

Hara, H. 1971. The Flora of Eastern Himalaya. Second Report, The University of Tokyo Press, Japan.

Hooker, J. D. 1872-1897. The Flora of British India. Vols. 1-7. L. Reeve \& Co., Kent, London. Hooker, J.D. \& Thomson, T. 1855. Flora Indica. Vol. 1. London.

Ikeda, H. Kurosawa, T. \& Ohba, H. 2008. Chromosome numbers and karyomorphology of three species of the genus Euphorbia L. (Euphorbiaceae) in the Sikkim Himalaya. Journal of Japanese Botany 83: 295 - 300

Maity, D., Maiti, G.G. \& Chauhan, A.S. 2018. Flora of Kanchenjunga Biosphere Reserve, Sikkim. Botanical Survey of India, Kolkata. Pp. 1 - 834

Noltie, H. J. 2000. Contributions to the Flora of Bhutan: The Monocotyledons. Royal Botanic Garden, Edinburgh.

Noltie H. J. 1996. Report of RBG Edinburgh Expedition to Northern Sikkim 1996 (EENS). Royal Botanic Garden, Edinburgh.

Ohashi, H. 1975. The Flora of Eastern Himalaya. Third Report, University of Tokyo, Japan. 